Björn-Olav Dozo \& Daphné De MARnefFe

Université de Liège

\title{
Réseaux et souvenirs littéraires : le cas d'André Fontainas
}

\section{Introduction}

manière thématique, évoquant successivement les revues symbolistes, ses débuts dans la vie

Mes Souvenirs du symbolisme ont été rédigés littéraire, les aînés qui l'ont marqué, les lieux de entre novembre 1924 et novembre 1925 par sociabilité littéraire (Librairie d'Art indépendant, André Fontainas (1865-1949), poète français Banquet Moréas, réunions et promenades du d'origine belge, qui vécut à Paris tout en cultivant soir). Il s'intéresse aux figures non littéraires du les amitiés nouées à Bruxelles dans sa jeunesse mouvement (musiciens, peintres, sculpteurs) et et servit de « trait d'union » entre les symbolistes au théâtre. Travaillés une quarantaine d'années belges et français. Auteur de plusieurs recueils après les faits, ces souvenirs — forcément (dont Sang des fleurs, 1889 ; Les Vergers parcellaires et subjectifs - proposent une vision illusoires, 1822 ; Les Estuaires d'ombre, 1895), parfois inégale du mouvement symboliste, mais ce poète discret fut aussi critique littéraire ${ }^{1}$ et ils ont le mérite de « rendre avec précision critique d'art (notamment au Mercure de France l'image d'un passé [plutôt que de] peindre un de 1890 à 1911), essayiste et traducteur de tableau feutré par le temps ${ }^{4} \gg$. Fontainas était certains grands écrivains anglais (Keats, Poe, d'ailleurs conscient du caractère incomplet et Shelley, Meredith, Swinburne). Reconnu par ses partiel de ses souvenirs, comme en témoignent contemporains ${ }^{2}$ et considéré comme un les premier et dernier chapitres de son livre, qui « témoin perspicace plutôt qu'un participant contiennent une sorte de commentaire passionné ${ }^{3} \gg$ du symbolisme, il se fit enfin le autocritique de l'entreprise ${ }^{5}$. mémorialiste du mouvement.

Nous proposons d'analyser le réseau

Comme l'indique le possessif du titre de son littéraire symboliste franco-belge, tel qu'il se ouvrage, Fontainas plonge dans ses souvenirs donne à lire dans les Souvenirs d'André personnels du symbolisme, dans le but de Fontainas, en abordant la question sous deux « tenter non pas une histoire ou un historique, angles d'approche complémentaires. Le mais une sorte d'esquisse à larges traits de ce «réseau » est un «système, non institutionnel moment littéraire » (p. 17). Il aborde le sujet de mais stable, de relation et de communication

Voir notamment les «Essais de Fontainas sur les lettres belges », republiés par Carmen Licari et Anna Soncini FratTa dans André Fontainas et ses amis belges. Rome, Bologne, Leo S. Olschki Editore, coll. Quaderni di Francofonia, VII, 1994, pp. 183-248

Comme en témoignent les critiques (de Giraud, Demolder, Eekhoud, etc.) reproduites par Carmen Licari et Anna Soncini Fratta (op. cit., pp. 249-268) et les nombreuses « lettres inédites » de 1889-1948 publiées dans le même ouvrage.

Fontainas (André), Mes Souvenirs du symbolisme. Bruxelles, Labor, coll. Archives du Futur, 1991, p. 17 (première édition: Paris, Revue Critique, 1928). Les paginations notées entre parenthèses à la suite des citations renvoient à cet ouvrage, dans l'édition de 1991

Sonctni Fratta (Anna), « Préface »a Fontainas (André), Mes Souvenirs du symbolisme, op. cit., p. 13. Premier chapitre : «Pourquoi j'écris ces souvenirs. Qu'est-ce que le symbolisme ?»; chapitre X : « Ce que je voulais faire dans ce livre. Mes omissions, Il libra della mia memoria». entre divers acteurs de la vie littéraire ${ }^{6} \gg$. Dans une première approche rhétorique, nous verrons comment Fontainas définit le symbolisme et comment (en quels termes) se dit le réseau. Dans une seconde approche narrative et schématique, nous prendrons appui sur le récit que fait Fontainas de sa propre entrée en littérature, pour reconstituer une partie isolable du réseau symboliste. Pour cette tentative de formalisation du réseau, nous nous inspirons de l'analyse structurale des réseaux utilisée en sociologie.

\section{Un symbolisme au croisement}

\section{de différents réseaux}

L'impression générale que les Souvenirs donnent du mouvement symboliste est celle d'une grande nébuleuse hybride et chaotique, dans laquelle il est difficile de mettre de l'ordre. Ces extraits mettent en évidence un rejet du Fontainas dresse une esquisse de ce que devait principe même des écoles littéraires et une être ce mouvement pour ses contemporains, dans revendication individualiste très affirmée. Ce ses nuances et sa complexité. Il s'agira de voir mouvement d'affranchissement par rapport à en quels termes il en parle : nous analyserons l'influence des écoles débute dans les revues, d'une part le type de définition — restreinte ou vers 1885 , sans qu'il soit possible d'identifier extensive - qu'il emploie et d'autre part les clairement une «première publication» champs lexicaux mobilisés.

symboliste ${ }^{7}$. Notons que le refus des écoles

Malgré l'éclatement thématique de son n'implique pas pour autant une négation de la propos, Fontainas livre par petites touches une valeur de leurs représentants. Le discours de définition assez précise du symbolisme. Ce Fontainas met en évidence les liens d'admiration, mouvement, qui «ne présente aucun des de fréquentation et d'influence ${ }^{8}$ qui unissaien caractères indispensables à constituer une les symbolistes à leurs aînés. En conséquence école » (p. 19) - dans la mesure où il n'y a pas de cette imbrication étroite entre les générations, de chef, pas de doctrine, pas de manifeste il est difficile d'isoler dans le texte des Souvenirs symbolistes - se définit « négativement », dans un « réseau » d'écrivains purement symbolistes, un rapport d'opposition à la logique du système ce que la seconde approche que nous proposons littéraire qui le précède : permettra de reconstituer partiellement.

Un autre indice de la définition « en creux »

Il ne m'est possible de discerner dans du mouvement est 1'absence de contenu l'ensemble du groupe symboliste qu'un programmatique de l'étiquette «symboliste » et

6 VAILlant (Alain), «Réseau et histoire littéraire : de la sociologie à la poétique », dans L'Analyse des réseaux (littérature, sociologie, histoire), actes du colloque du « CIEL », Liège, 20-21 mars 2003, à paraître.

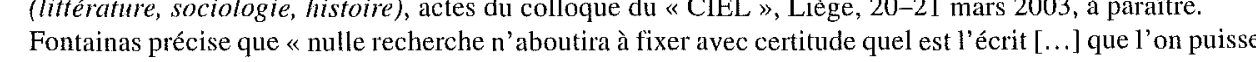
légitimement considérer comme [...] la première publication du symbolisme» (p. 25) et que «le germe symboliste a levé jusqu' au tréfonds de publications strictement parnassiennes » (p. 26) : dès 1875, « on [y] découvre des vers, des proses qui s'écartent de l'orthodoxie parnassienne, qui se dérobent aux tendances du réalisme et du naturalisme » (p. 26). Il cite alors les revues le Parnasse contemporain, La République des Lettres, la Revue du monde nouveau et le Spectateur (p. 26), dans lesquelles publièrent très tôt Mallarmé, Verlaine, Villiers ou Charles Cros. En 1886 paraissent Le Symboliste, Le Décadent et La Vogue de Léo d'Orfer, qui publie des vers libres de Kahn, Laforgue, Moréas. (Pour la liste complète des revues citées, voir pp. 26-29.)

«Ces grands écrivains [Hugo, Baudelaire, Leconte de Lisle, Léon Dierx, Barbey d'Aurevillyl, à leur insu, même en dépit d'eux, ont exercé sur les destinées du symbolisme une influence, bien qu'on soit porté à la négliger ou qu'on la conteste [...] Il n'est pas exact, non plus, d'affirmer que l'ascendant de Mallarmé et de
Verlaine, dont on fait, en même temps que Baudelaire, nos précurseurs exclusifs, se soit étendu également sur tous les poètes de nos générations [...]. » (p. 53) 
l'impossibilité de déterminer une esthétique La voix de Mallarmé séduit et suscite une sorte communément partagée (fût-ce celle du « vers- de vénération religieuse («parole libre s):

transfiguratrice », «muette dévotion», " incantation multiple»). Guide charismatique

Que n'a-t-on point allégué encore du mais pas doctrinaire, Mallarmé « renseigne » vers-librisme, de l'école du vers-libre ? Or, sans enseigner, sans imposer ses vues, sans c'est surtout là qu'il n'y a pas eu, qu'il ne chercher à convaincre. Les rapports pouvait y avoir école. Qui s'y fût targué interpersonnels se déclinent d'ailleurs sur le d'avoir édicté un système et prêché des mode de la fraternité et non de la relation entre initiés ? On ne sait pas au juste où le vers- maître et disciples. Par exemple, à propos de sa libre a pris naissance et lequel de ses artisans première rencontre avec Mallarmé, Fontainas se en a usé le premier. [...] La quête du souvient d' $d^{\prime}$ un accueil tout de suite fraternel ; symbole, l'adoption du symbole en tant que la chaleur réservée et cordiale de sa poignée de mode d'expression lyrique, peut-on main au départ» (p. 39). Au niveau de la prétendre, comme on l'a tenté, que c'est par désignation du mouvement, l'emploi de termes là que nous nous ressemblions? Ce fut le vagues traduisant une réalité peu structurée est prétexte dont se saisit pour nous répudier de constant

l'école romane. Pourtant il est bien exact que, à l'exception peut-être de Mallarmé, personne, dans les débuts du moins, ne s'était fait une règle ou une conception quelconque du symbole en littérature. (pp. 21-23)

La raison du regroupement de ces jeunes écrivains sous une même appellation est donc purement pratique : il s'agit d'« être unis pour affirmer chacun son individualité, pour s'assurer un champ propice, au milieu du concours trouble Rejetant l'idée de «former école », Fontainas et confus des appétits satisfaits et des glorioles parle de «susciter un mouvement [...], un égoïstes » (p. 24), non pas de s'enrégimenter sursaut de renouveau ». Ailleurs on recense les dans un système contraignant.

expressions de «moment littéraire » (p. 17)

Il est d'ailleurs intéressant de voir en quels $d^{\prime}$ « ensemble » ou de « grand concours de jeunes termes est présentée la figure de Mallarmé, gens » (p. 18, 21), de « groupement (p. 128) principal élément fédérateur du groupe :

de « mouvement » (p. 23, 25, 128), de « famille intellectuelle» (p. 129) et de «grande,

Le mardi soir, quand autour de Mallarmé nombreuse famille d'influences saines » (p. 59). on se pressait à l'écouter, chacun perdait le Dans tout le texte, il n'y a qu' une occurrence de sentiment d'être différent. [...] Ceux qui «brigade symboliste » (p. 113), désignation n'ont point joui de ces réunions-là peuvent- singulièrement inappropriée à son objet, dans la ils imaginer une parole transfiguratrice à ce mesure où les symbolistes semblent résister à point, et à propos de tout sujet, comme tout «embrigadement».

ingénument cérébrale, selon le délice de $s a$ Au passage, pointons la première ligne de voix timbrée, nuancée, sourde un peu, l'extrait ci-dessus ( Restreignons 1a enveloppante et assurée ? [...] La foule se signification de ce terme à ceux-là seulement »), représente un Mallarmé doctrinaire et qui qui fait explicitement référence à une acception enseigne, où nous assistions, emplis d'une restreinte, spécifique, du terme « symbolisme ». muette dévotion, à l'éclosion incessante Malgré son effort pour définir précisément son d'une fleur d'âme. [...] Mallarmé sujet, Fontainas a plusieurs fois recours à une n'enseignait pas ; il renseignait. Son dessein acception très large de ce terme. En début comme ne consistait ni à convaincre ni à éblouir. Sa en fin d'ouvrage, le symbolisme est assimilé à voix pliée aux souplesses des syllabes élevait l'ensemble de la « grande littérature » ${ }^{9}$. Il nous une incantation multiple. (p. 114) [nous semble que ce procédé de «dilution du sens » a soulignons] mouvement et celle de son extension d'usages », sont discrètement assimilés aux géographique ou nationale : dans son acception Français. Or, lorsque l'on considère extensive, le symbolisme est intemporel (donc spécifiquement les symbolistes belges, les "éternel») et universel. On retrouve la même considérations sociologiques de Fontainas sont "eternel symbolisme dans les passages où Fontainas traite souligné la grande «cohésion sociale du de la composition du groupe symboliste: symbolisme belge ${ }^{11} \gg$ et ses effets positifs sur la durée du mouvement.

D'où venait ce grand concours de jeunes Cette «confusion» entre symbolistes hommes ainsi épris, en même temps, d'un français et belges est quasi constante dans les idéal pur et vaste? Inconnus les uns aux Souvenirs d'André Fontainas. De manière autres, éloignés par des différences infinies générale, il ne précise pas la nationalité des de caractère, d'origine, d'éducation, de auteurs dont il parle ${ }^{12}$. Il n'aborde d'ailleurs tendances spéculatives et esthétiques, de race jamais la question de l'apport spécifique des aussi, ils se rapprochaient à cause de ce qu'ils symbolistes belges au mouvement et néglige les pes Belges 13 pressentaient en eux de commun, d'analogue anecdotes ne concenna que des Belges en sincérité, en spontanéité profonde, en Lorsque Fontainas parle du symbolisme, il fai élans ingénus vers [...] le domaine de l'art, référence à un tout rassemblant Français et le domaine de la pensée. [... À côté de Belges. Il est vrai que lui-même vivai le domaine de la pensée. grand nombre provenaient [...] d'île-de- française et qu'il a longuement travaillé à créer Grand osmose » entre les écrivains des lointaines ou de contrées plus proches, deux nationalités ${ }^{14}$. On ne peut cependant lointaines ou de contrées plus proches, deux nationalités ${ }^{14}$. On ne peut cependant
Quelques-uns enfin, outre les Belges et les oublier qu'en tant que critique, Fontainas était Suisses, si voisins de langue et d'usages, conscient de l'existence d'un appartenaient à des nationalités étrangères « symbolisme belge » qu'il a plusieurs fois pris [...]. (p. 18) pour objet ${ }^{15}$. Quoi qu'il en soit, cette assimilation a plusieurs effets.

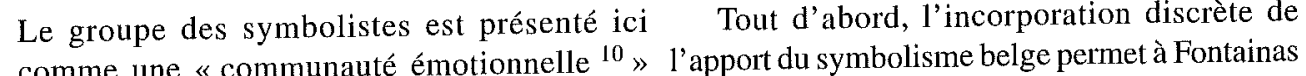
comme une «communauté émotionnelle ${ }^{10}$ " l'apport du symbolisme belge permet à Fontainas soudé dans un mémé (sociale, culturelle, acception restreinte) a perduré jusqu'au moment intellectuelle, nationale). On remarquera que les où il rédige ses Souvenirs, au milieu des années Belges (et les Suisses), « si voisins de langue et vingt :

9 «À travers les siècles, dans toute l'étendue de l'histoire littéraire, dans toutes les régions du monde où s'est

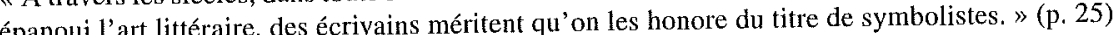

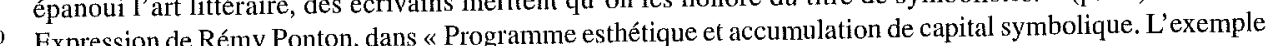
du Parnasse ", dans Revue française de Sociologie, XIV, 1973, p. 209.

ARON (Paul), « Pour une description sociologique du symbolisme belge », Le Mouvement symboliste en Belgique. Sous la direction d'Anna Soncini Fratta. Bologne, Clueb, coll. Beloeil : atti del Centro studi sulla letteratura belga di lingua francese, 1990, pp. 61 et 67. Soncini FratTa (Anna), « Les Symbolistes belges entre chronique et histoire littéraires », dans André Fontainas et ses amis belges, op.cit., p. 26.

12 Le chapitre sur sa propre entrée en littérature fait exception. On y trouvera quelques éléments sur l'émergence Le cha du symbolisme belge. Fonain de Provenance de Brelles ou de

qu'il connaissait et classe les réponses reçues selon leur provenas dans la liste de ses «omissions»- les

Par exemple, Fontainas ne mentonne pas du tout - mene pas das la liste de ses « omissions »- - les « dîners belges » à Paris, dont on retrouve pourtant des traces dans sa correspo

Soncin Fratta (Anna), André Fontainas et ses amis belges, op.cit., p. 93).

Sur cette question, consulter LicA

en Belgique, op.cit., pp. 71-83. Carmen Licari (Ibid., p. 77) liste les études pano, notamment, il publie « Les débuts et les tendances du conferences de Fontainas sure spécificités du symbolisme belge (dans La Revue des Lettres et des Arts, juillet 1909, pp. 473-491). 
Au surplus, ce moment, s'il a commencé structure stable), il en effet difficile d'éviter de s'ébaucher aux environs de 1885, ou l'écueil de la complète dissolution du sens de même de 1890, ne me fait pas l'effet de s'être cette «étiquette »dans une acception plus large. éteint jusqu'à présent [novembre 1925], en qui assimile le symbolisme à l'ensemble de la dépit d'éclipses ou d'offuscations partielles, littérature. Une anecdote nous paraît illustrer un et bien qu'on se soit à plusieurs reprises hâté autre aspect de la difficulté posée par ce type de d'en proclamer la mort définitive et l'oubli définition « en creux ». Par son essai $L a$ total. (p. 17)

Litterature de tout à l'heure - auquel Fontainas consacre sept pages de ses Souvenirs En toute sincérité, en considérant l'ensemble publication qui «força l'attention » et fut lue symboliste franco-belge, Fontainas peut soutenir comme «le credo d'une génération », l'inconnu en 1925 que celui-ci n'est pas encore éteint : la Charles Morice devint « soudain presque l'âme "crise des valeurs " et la polémique anti- du mouvement, sa conscience, son chef » (p. 28) symboliste des années 1895-1900 est propre au Comme il ne prétendait s'imposer à personne, il symbolisme français ${ }^{16}$. Le symbolisme belge, «inspira confiance » et « on se resserra autour quant à lui, reste «actif sur la scène littéraire de lui » (p. 29). En 1891, il conçut le projet jusqu'à la veille de la guerre 1914-1918 » et dix d'imposer le symbolisme au théâtre, avec l'aide ans plus tard, en 1924, paraissent encore de de Catulle Mendès. Cette tentative se solda par belles cuvres symbolistes, dont les recueils de un échec, d'autant plus retentissant que l'on avai Max Elskamp ${ }^{17}$. De la même manière, dans le cru et espéré dans la réussite du projet de celui chapitre IX intitulé « Le symbolisme et la vie », qui s'était " haussé à la stature d'un chef » Fontainas argumente contre l'accusation qui fut (pp. 97-98). Ici, le discours de Fontainas traduit faite au symbolisme (français) de «tourner le la tentation de voir en Charles Morice un dos à la vie et de se réfugier dans le rêve ${ }^{18}$ », en « leader providentiel» du mouvement (« âme du s'appuyant principalement sur l'exemple (belge) mouvement », «sa conscience », « son chef », de Verhaeren ${ }^{19}$ " haussé à la stature d'un chef», etc.). C'est

Un bref détour par le thème du théâtre (traité assez curieux, dans la mesure où Fontainas a bien au chapitre X des Souvenirs) nous permettra résisté ailleurs à la tentation de structurer le d'illustrer les différents points que nous avons mouvement par l'identification d'un «chef » et abordés. d'une « doctrine » symbolistes : comme dit plus

A propos de la définition du mouvement, haut, il a d'une part insisté sur l'impossibilité nous avons vu que le groupe est dépourvu de définir une esthétique commune et d'autre d'éléments fédérateurs (pas de chef, pas de part, il a précisé en quoi Mallarmé, élément doctrine, pas de manifeste), si ce n'est fédérateur, ne doit pas être considéré comme un l'opposition commune à l'idée d'école et « chef ». Cette anecdote nous paraît illustrer la l'adhésion à un même idéal de pratique libre de difficulté à évoquer un mouvement sans avoir 1'art. Le fait que le symbolisme se définisse « en recours, à un moment ou un autre, aux points de creux » implique que les points de repère repère traditionnels, quitte à hisser à la position traditionnels restent des «cases vides ». Nous de «chef » quelqu'un qui se montre incapable avons vu Fontainas hésiter entre une acception d'assumer ce rôle.

restreinte et une acception large du symbolisme. Par ailleurs l'exemple du théâtre nous permet Dans la mesure où il n'y a pas de définition d'illustrer la complexité du réseau des écrivains «positive » du mouvement (en référence à des symbolistes. Nous avons déjà signalé son éléments précis qui proposent un contenu et une imbrication étroite avec les générations

16 Michel Otten rappelle l'éphémère existence de l'école parisienne symboliste, de 1885 à 1895 (« Originalité du symbolisme belge », dans Le Mouvement symboliste en Belgique, op.cit., p. 16, p. 22).

17 Ibid., p. 16, p. 25

18 Ibid, p. 22, où Michel Otten rappelle les critiques qu'Adolphe Retté, Camille Mauclair et des Jeunes Naturistes adressèrent au mouvement symboliste français.

19 Paul Aron a expliqué comment les symbolistes belges - qui par leur formation étaient en phase avec la vie politique et sociale de leur époque et partageaient une «idéologie libérale-socialiste [...] porteuse d'une vision optimiste appliquée à l'évolution historique» - se sont mieux adaptés au «tournant ver l'optimisme $»$ de la fin du siècle, comme en témoignent différents recueils poétiques dont le titre fait référence à la «Vie ». (Aron (Paul), op.cit., pp. 66-67) précédentes (réseaux parnassiens, naturalistes). Analyse d'un réseau Les Souvenirs de Fontainas offrent aussi un dans les Souvenirs du symbolisme précieux témoignage des nombreux rapports entretenus par la littérature avec les autres arts. poètes, les musiciens (Wagner ${ }^{20}$ mais aussi différemment : l'objectif est de formaliser et de Debussy, Vincent d'Indy, Ravel, Fauré) et les schématiser les relations entre Fontainas et les peintres ${ }^{21}$ (dont Seurat, Gauguin ${ }^{22}$, les nabis). différentes personnes telles qu'il les mentionne Le théâtre est par excellence à l'intersection de au fil du récit. Cette formalisation s'inspire ces différents réseaux. Par exemple, c'est librement de la méthode d'analyse structurale Gauguin qui créa décors et costumes pour des réseaux utilisée depuis longtemps en l'événement de Charles Morice (p. 97); les sociologie ${ }^{24}$. Cette méthode utilise la notion de nabis firent des décors pour le Théâtre d'Art et réseau comme un instrument explicatif, et pas le Théâtre Libre (p. 68) ; la pièce Pelléas et comme objet à expliquer. Nous ne prenons donc Mélisande a survécu comme drame lyrique, plus le réseau comme un objet qui se dit et qu'on grâce à la musique de Debussy (p. 103) étudierait en tant que tel (voir supra) mais plutôt

Enfin, le thêâtre illustre aussi l'apport belge comme un outil qui fait parler un autre objet au mouvement symboliste. En finale du chapitre la représentation qu'a Fontainas de ses consacré à ce sujet, Fontainas conclut à l'échec relations - grâce à la constitution des nœuds général du symbolisme au théâtre. Quelques du réseau et à la formalisation schématique de «réussites » Sont cependant citées : celles de leurs relations. Nous proposons donc une analyse Rodenbach (Le Voile) et d'Albert Samain réticulaire de la représentation qu'a l'auteur de (Polyphème). Au programme de l'ensemble fini de ses relations, que l'on pourrait « l'événement théâtral » dirigé par Charles appeler métaphoriquement son « réseau 》.

Morice, principalement composé de poèmes, il Une formalisation schématique vise, selon n'y a que trois «pièces " qui ressortissent la méthode classique de l'analyse structurale des véritablement au genre théâtral (Les Uns et les réseaux, à la complétude du nombre de nœuds. Autres de Paul Verlaine, «chanson alternée», Si cette complétude est difficilement accessible en un acte en vers ; Chérubin de Charles Morice, pour des relations interpersonnelles dans la drame bâclé qui coula son auteur ; et L'Intruse réalité, elle est tout à fait envisageable en ce qui de Maeterlinck, en un acte). La seule « exception concerne les souvenirs écrits (qui sont de fait remarquable » du chapitre est la persévérance limités au texte et donc à la volonté de l'auteur). de Maurice Maeterlinck (p, 103). Nous Chaque nom cité est susceptible de constituer n'insisterons pas sur le fait que le théâtre un nœud. Pour obtenir le «bout de réseau » qui symboliste est presque exclusivement belge ${ }^{23}$. joue le rôle le plus cohérent et significatif dans

Il semble donc qu'en considérant les les Souvenirs du symbolisme, nous avons tenté symbolismes français et belge comme un tout, d'identifier quels étaient les personnes les plus Fontainas donne une certaine ampleur au souvent citées. Cette méthode quantitative mouvement qu'il décrit, tant au niveau de son permet de dégager un groupe que l'on retrouve extension générique (de la poésie au théâtre) que tout au long du texte. Les personnes de ce groupe diachronique (longue durée du symbolisme fonctionnent comme des motifs relationnels belge, en relation avec sa capacité à s'adapter récurrents, qui permettent à Fontainas de aux thématiques « optimistes » du tournant du structurer le texte et de lui donner une cohérence siècle).

interne. La mise en récit de la prise de position

20 Voir l'exemple de la Revie wagnérienne d'Édouard Dujardin, 1885, p. 62.

21 Voir notamment toutes les mentions de Joris-Karl Huysmans dans À Rebours (la Salomé de Gustave Moreau, Odilon Redon, Puvis de Chavannes, etc.).

22 Les Lettres de Gauguin à André Fontainas furent publiées par ce dernier en 1921 (Paris, Librairie de France).

23 Voir l'intéressante hypothèse de dysfonctionnement générique de Paul Aron, qui explique comment les Belges ont adopté des genres peu fréquentés par les auteurs français, dont le théâtre (Aron (Paul), op.cit., p. 62).

24 CLAISSE (Frédéric), « De quelques avatars de la notion de réseau en sociologie », dans L'Analyse des réseaux (littérature, sociologie, histoire), op.cit., à paraitre. 
de chacun des membres dans les différents lieux prennent une importance considérable dans les de sociabilité symbolistes est complémentaire Souvenirs, et au moins l'un d'entre eux (bien d'une esquisse du mouvement. souvent plusieurs) est cité par Fontainas à chaque

Le premier acquis de la méthode d'analyse occasion thématique ${ }^{27}$ (revues belges et par dénombrement et schématisation réticulaire françaises, Mardis de Mallarmé, Librairie de est l'identité des membres de ce groupe : il ne l'Art indépendant, banquet en l'honneur de s'agit pas des personnes-phares du symbolisme, Moréas, tentative d'événement théâtral de canonisées par l'histoire littéraire, mais des amis Charles Morice).

de jeunesse de Fontainas. En surplus des À Bruxelles, Fontainas suit les cours de la souvenirs communs à tous les symbolistes, celui- faculté de droit. Il participe à la fondation d'une ci écrit l'histoire de son propre réseau, dont il revue, La Basoche, "revue artistique et souligne les activations dès qu'il le peut, en littéraire $»$. Petite revue d'étudiants, marge de chaque grand événement symboliste. ambitionnant seulement d'exister et de Le nombre d'occurrences des noms de ses amis rassembler des ouvres littéraires de toutes indique qu'ils occupent une place importante à origines, La Basoche tient seize mois. Après ses yeux dans le mouvement symboliste. La avoir énuméré quelques Belges qui y auraient schématisation se focalisera donc sur les fait leurs premières armes, Fontainas rapporte relations abondamment soulignées par que "la collaboration qu'y apportaient des Fontainas. Reste maintenant à suivre le fil Français était particulièrement remarquable, narratif des Souvenirs du symbolisme pour en avec les noms de [...] Rodolphe Darzens, René dégager les moments importants du mouvement, Ghil [...], Stuart Merrill, Ephraïm Mikhaël, dans l'optique de Fontainas.

Pierre Quillard [...] ». On retrouve donc l'équipe L'entrée en littérature de Fontainas date du du lycée au grand complet ${ }^{28}$.

lycée : «Mes camaraderies littéraires se D'autres revues, à la renommée plus fortifièrent durant cette période, et s'enhardirent. importante, sont à peine abordées dans les J'en avais cimenté de robustes qui restèrent Souvenirs: La Jeune Belgique, La Wallonie, Le inébranlables jusqu'à la mort. » (p. 40) Il Coq rouge... En expliquant en quelques mots s'interroge sur les raisons qui ont poussé des l'atmosphère qui règne aux rendez-vous du soir « rhétoriciens » à s'intéresser à lui, qui n'était de La Jeune Belgique, Fontainas souligne le lien « qu'en seconde ». Ses amis du lycée Condorcet, qui existe entre les poètes belges expatriés et le aux réunions ${ }^{25}$ desquels il assiste tous les jeudis groupe de ses amis ${ }^{29}:$ « Rodenbach déjà s'était dans une petite chambre d'hôtel, sont pourtant établi à Paris où séjournaient pour un temps les poètes qui l'accompagneront toute sa vie : Maeterlinck, Grégoire Le Roy. Ils y collaboraient Ephraïm Mikhaël (Georges Michel), Rodolphe à la Pléiade de mes vieux camarades Darzens, Darzens, Pierre Quillard, René Ghil (René Mikhaël et Quillard [...] »(p.44) De même, Guilbert) et Stuart Merrill ${ }^{26}$. Ces cinq amis quand il signale la revue d'Albert Mockel,

25 À propos de ces réunions, voir LefrEkRE (Jean-Jacques), « Lettres inédites d’Ephraïm Mikhaël », dans Histoires littéraires, Juillet-août-septembre $2003, \mathrm{n}^{0} 15, \mathrm{p} .84$.

26. À six, ils fondent une revue littéraire, Le Fou, dont Fontainas ne mentionne pas l'existence. Pour une étude de cette revue, voir l'article de VANWELKENHUYZEN (Gustave), « Un trio de revues », dans Revue de l'Université de Bruxelles, 1974, III-IV (« Le mouvement symboliste en littérature »), pp. 318-334. Voir aussi Lefrère de Bruxelles, 1974, III-IV (« Le moun-Jacques), op. cit., pp. 84-85.

27 Des le premier chapitre, lorsqu'il est question de l'admiration pour les anciens, les noms de Quillard et Merrill apparaissent : «Or il n'y eut jamais à Victor Hugo d'admirateurs plus fidèles que Henri de Régnier par exemple, que Pierre Quillard, Stuart Merrill, Pierre Louÿs ou moi-même, » (p. 20)

Lefrère confirme le rôle de passeur de Fontainas : «À ses débuts, La Basoche comptait parmi ses collaborateurs presque autant de Français que de Belges. [...] Fontainas [...] avait introduit ses amis parisiens dans la revue [...] ». (Lefrè̀re (Jean-Jacques), op. cit., p. 88)

29 A ce propos, Fontainas passe complètement sous silence son propre rôle d'intermédiaire. Un des premiers Belges à arriver à Paris (en 1889, un an après Rodenbach), Fontainas aida La Jeune Belgique de Valère Gille à trouver des appuis dans la capitale française (cf. SONCINI FRATTA (Anna), « Les symbolistes belges, Gille à trouver des appuis dans la capitale française (cf. SONCINI FRATTA (Anna), « Les symbolistes belges,
entre chronique et histoire littéraires », op. cit., pp. 37-39). Les nombreuses lettres éditées par Carmen entre chronique et histoire littéraires $»$, op. cit., pp. 37-39). Les nombreuses lettres éditées par Carmen
Licari et Anna Soncini Fratta (dans André Fontainas et ses amis belges. Lettre inédites 1889-1948) illustrent Lieari et Anna Soncini Fratta (dans André Fontainas et ses amis belges. Lettre inédites
bien la relation d amitié et de soutien qui unissait Fontainas aux écrivains belges.
Fontainas ne met en évidence que la de France et au banquet Moréas. Et c'est Hérold collaboration de «fidèles aux vers réguliers, - dont Fontainas épouse la sœur, Gabrielle, en comme Severin ou comme Quillard » (p. 45). La $1890^{34}$ — qui introduisit ce dernier au Mercure Wallonie est d'ailleurs traitée de manière de France (p. 46).

extrêmement elliptique dans ses Souvenirs, alors De même, Fontainas dut être poussé par ses que différents critiques insistent sur la grande anciens amis de lycée pour prendre part aux amitié qui liait Mockel et Fontainas et sur leur mardis de Mallarmé. Le contact s'était déjà établi correspondance abondante ${ }^{30}$ par correspondance, Fontainas ayant envoyé à

Après ces quelques remarques sur les revues Mallarmé sa première œuvre et ayant reçu en belges, Fontainas consacre plusieurs pages à la réponse : «Si vous vous égariez jamais par ici grande revue symboliste française, Le Mercure un mardi dans la soirée, je serais content de de France. «Il n'est point de réputation littéraire, causer. » (p. 39) Mais comme l'écrit Fontainas entre 1890 et la guerre de 1914, qui ne soit issue «Jamais je n'aurais osé m'y présenter si mon du Mercure ou qu'il n'ait pas contribué à très cher camarade Pierre Quillard, qui déjà y former: " (p. 47) Il la présente comme succédant fréquentait, ne m'eût fait honte de ma couardise à La Pléiade de Darzens ${ }^{31}$. Cela se justifie d'un et ne m'eût entraîné presque de force plutôt que point de vue esthétique, selon l'hypothèse de la par persuasion. » (p. 39)

filiation des revues défendue par Gustave Quillard - décidément partout ! - apparaî Vanwelkenhuyzen ${ }^{32}$, mais au niveau du encore dans deux lieux de sociabilité auxquels personnel de la revue, les intervenants sont Fontainas attache de l'importance : la Librairie différents : pris en main par Alfred Valette, Le de l'Art indépendant et le banquet en l'honneur Mercure intéresse de nombreux collaborateurs, de Jean Moréas. Le chapitre VI explicit dont les amis de jeunesse de Fontainas ne l'activité de cette librairie et la personnalité de constituent pas le noyau dur. Ils y sont tout de son patron, Edmond Bailly - pour qui « dè même impliqués relativement tôt: Fontainas qu'un livre se vend, c'est qu'il ne vaut rien » dresse une liste de collaborateurs, et mentionne (p. 76). On y trouve une liste des jeunes gen Quillard dès 1890 (juste après le premier qui fréquentaient et entouraient les aîné numéro) et Merrill en 1892.

présents. Quillard en fait partie. De plus, lorsque

Il faut remarquer la présence dans la liste de Fontainas donne des exemples de publication collaborateurs d'un dernier intervenant: André- de cette librairie, il cite trois ouvrages traduits Ferdinand Hérold, un autre camarade de lycée l'un de ceux-ci est le Livre des mystères de de Fontainas, qui participe, d'après Gustave Jamblique traduit par Quillard, et un autre es Vanwelkenhuyzen ${ }^{33}$, à la création de la revue L'Upanishad du grand Aranyaka, par Hérold Le Fou au lycée Condorcet. Sa présence dans (p. 76). Dans les Souvenirs suit immédiatement les Souvenirs est moins marquée en ce qui le compte rendu du banquet offert à Jean Moréas, concerne les revues des débuts - Fontainas le 2 février 1891, qui voyait se sceller « l'amiti mentionne tout de même qu'il lui envoie sa entre deux générations par la célébration d'u première œuvre, Le Sang des Fleurs —, mais il idéal commun, le culte de la poésie » (p. 77) fait aussi partie des lycéens de Condorcet et on Quillard et Hérold sont là aussi en bonne place le retrouve cité pour sa participation au Mercure parmi la génération montante.

30 LiCaRI (Carmen), «André Fontainas parmi les siens", dans André Fontainas et ses amis belges, op.cit, p. 2. Dans le même ouvrage on trouve une étude de Fontainas sur « Le rôle de la Wallonie dans le mouvement symboliste » (Monde Nouveau, 15 septembre 1924, pp. 19-29), où il affirme que « [la Wallonie est] la revue qui, véritablement, en Belgique, soutint et propagea l'effort symboliste » (LiCArI (Carmen) et SoNCiNı FRATTA (Anna), «Essais de Fontainas sur les lettres belges », op.cit., p. 238).

31 En fait, Binn' La Merce Brinn' 'Gaubas : « Cele entre mai et octobre 1889), mate sur le verso de couverture, première mouture du Mercure de France, dont les numéros initiaux portent de

la mention "La Pléiade, $2^{\mathrm{e}}$ année". 》 (Lefrère (Jean-Jacque
Voir VAnwhlkenhuYzen (Gustave), op.cit., pp. 331-332.

33 Ibid., p. 318

34 LICARI (Carmen), «André Fontainas parmi les siens », dans André Fontainas et ses amis belges, op.cit., p. 6, n. 16. 
Ces nombreux exemples n'ont pas épuisé Fontainas reste pourtant extrêmement discret l'abondance des mentions des noms des lycéens sur la mise en valeur de ses premières amitiés de Condorcet. Pour reprendre l'anecdote de la littéraires : cette prédominance de ses anciens première partie concernant le théâtre, il est camarades de lycée se dit presque malgré lui. amusant de constater que c'est encore par Seul Mikhaël, mort jeune, est mis en évidence Quillard que Fontainas, alors à l'étranger, explicitement ${ }^{35}$.

apprend le désastre de la «manifestation d'art » Nous sommes maintenant en mesure de de Charles Morice :

constituer un schéma reprenant les noms de personnes les plus citées (le nombre

Je [Quillard] regrette vivement que le d'occurrences d'un nom constituant ainsi notre succès en ait été si manifestement nul : il me premier critère de sélection pour le schéma) et devient plus difficile de dire tout le mal que les lieux de sociabilité (revues ou lieux de j'en pense - et cependant, (ajoutait-il) il faut rencontre) les plus importants à ses yeux (la le dire, ne fût-ce que pour nier la solidarité place réservée dans le livre à des lieux privilégiés des poètes nouveaux, non point avec un mentionnés en titre de chapitre devenant notre homme de talent méconnu, mais avec un second critère de sélection). médiocre dramaturge. (p. 98)

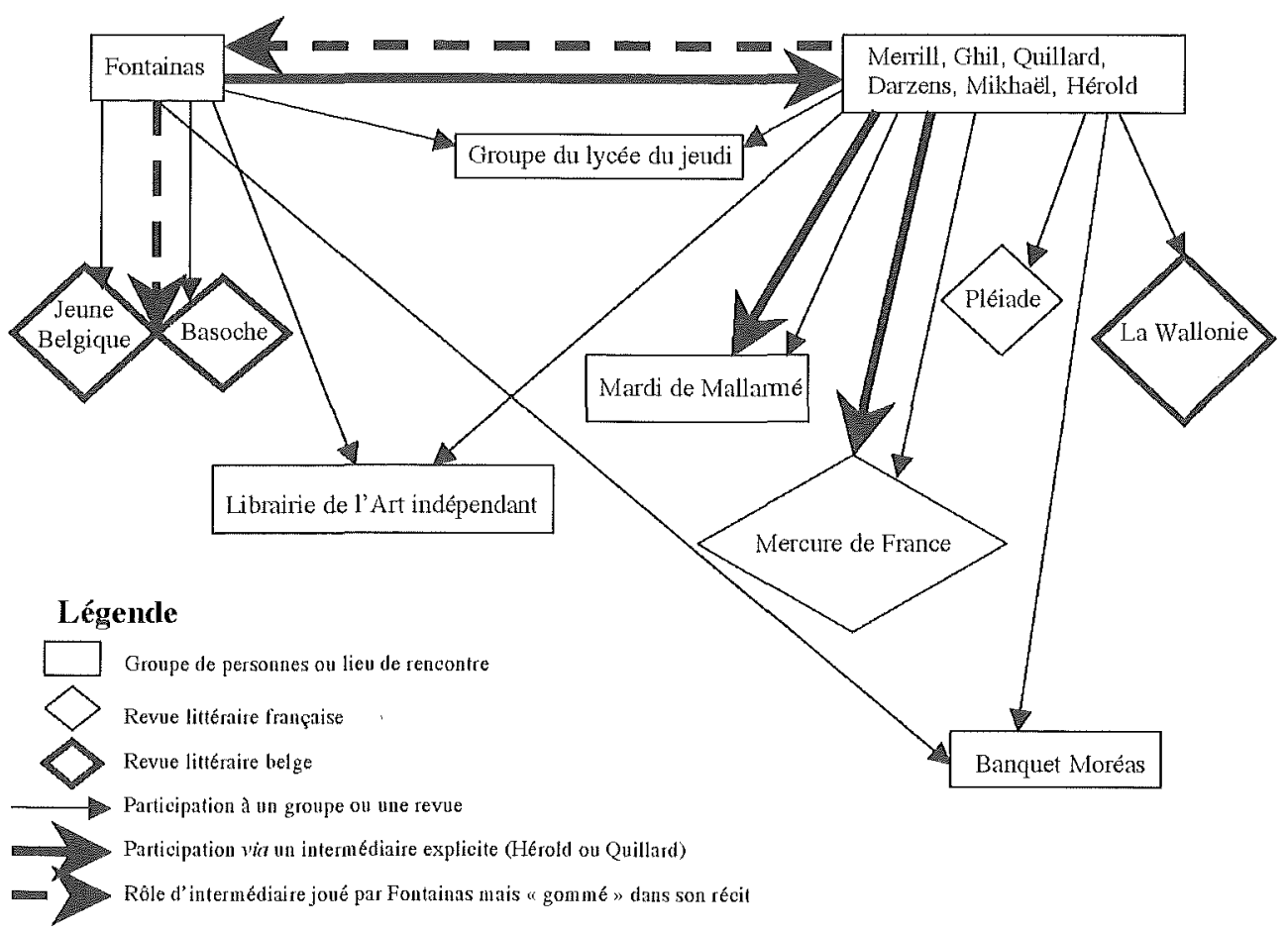

Ce schéma appelle plusieur's remarques, qui Fontainas ne fait explicitement aucune différence sont en fait des conséquences de l'identité des entre l'apport belge et l'apport français, mais le personnes les plus souvent citées. On aborde à réseau de relations le plus visible dans ses nouveau le problème de la nationalité du Souvenirs est un réseau exclusivement français, mouvement dont il a déjà été question dans la composé de ses amis d'adolescence qui première partie de l'article. Les remarques que l'accompagnent toute sa vie. le schéma suggère vont dans le même sens

35 Fontainas (André), op.cit., p, 124 : «Aussi, au long de ces pages, me suis-je particulièrement complu à rendre à des poètes morts très jeunes, à Mikhaël en premier lieu, un hommage que j'estime encore insuffisant. »Voir aussi pp. 80-84, les pages dédiées à Mikhaël. Sur ce poète, voir la récente bio-bibliographie de Lerrekre (Jean-Jacques), op.cit., pp. 81-113.
De plus, si Fontainas insiste et met bien en compte de ce que les Souvenirs d'André évidence sa dépendance vis-à-vis d'eux pour son Fontainas disent du symbolisme. Premièrement, introduction dans les lieux de sociabilité français, ce qui se dessine, tant à travers l'analyse des il passe complètement sous silence le rôle définitions du symbolisme que par le d'intermédiaire qu'il joue entre son groupe recensement des noms cités, est un objet d'amis de la première heure et les revues belges (mouvement ou réseau) complexe, aux contours — rôle que l'on connaît bien par ailleurs et qui flous : d'une part, une acception restreinte du est mentionné en pointillés sur le schéma. terme est confrontée à une acception plus large

Il faut aussi souligner que les seuls lieux de et les éléments de définition sont principalement sociabilité belges mentionnés par Fontainas sont «négatifs »; d'autre part, on s'aperçoit que les des revues: si les symbolistes publient noms retenus et abondamment cités par régulièrement en Belgique, ils vivent et se Fontainas ne sont pas ceux traditionnellement réunissent en France, seul pays où les lieux de mis en évidence par 1'histoire littéraire. On sociabilité existent réellement. Fontainas, en obtient donc une image d'un symbolisme accordant autant de place aux événements différent de celui des manuels, qui renvoie à français, inscrit le cour du mouvement en d'autres œuvres de référence (La Littérature de France, et ne laisse à la Belgique que le rôle tout à l'heure de Charles Morice) et à un d'une terre d'émergence ou — mais cela personnel littéraire habituellement occulté. n'apparaît pas dans le texte et ne peut être Deuxièmement, une analyse rhétorique comme compris que grâce à la mise en perspective de la une approche schématique du réseau mettent en durée du mouvement (voir supra) - de fin de évidence le rôle déterminant des amitiés et vie du mouvement symboliste. camaraderies littéraires: les relations

L'apport principal de l'analyse réticulaire de s'établissent sur le registre de la «fraternité » souvenirs littéraires, outre l'identification des (parfois doublé de celui de la "vénération » vispersonnes les plus souvent citées dans ces à-vis des précurseurs) et le réseau sous-jacent à souvenirs, reste la mise en avant de la travers tout le texte est celui des amis de jeunesse. représentation qu'a l'auteur de la pérennité de Troisièmement, lorsque l'on aborde les choses ses relations amicales de jeunesse. Cette d'un point de vue spécifiquement belge, il faut représentation prend corps par la mention de ces constater que dans ses Souvenirs, Fontainas fait noms dans ce que Fontainas considère comme œuvre de critique «français »: il incorpore les principaux lieux de sociabilité du discrètement l'apport belge dans sa présentation mouvement. Enfin, le schéma permet du symbolisme, sans faire de distinction entre d'embrasser d'un coup d' œil ces relations et ces les symbolismes français et belge ${ }^{36}$. De plus, le lieux. réseau dans lequel il s'intègre est principalement français : Fontainas ne rend que partiellement compte de son rôle d'intermédiaire entre Conclusion

Belgique et France, gommant tout ce qui

En combinant deux approches de l'analyse concerne la dépendance des Belges vis-à-vis de des réseaux littéraires, nous avons tenté de rendre lui.
36 Michel Otten rapporte que «si la France incorpore généralement les symbolistes belges dans le mouvement français, l'Allemagne, l'Italie, la Pologne ou la Russie ne les ont jamais confondus et [que] l'ensemble du mouvement est parfois appelé, en Europe, le symbolisme franco-belge » (OTrEN (Michel), op.cit., p. 16). 